\title{
Uma questão masculina: conhecendo possíveis entraves para a realização dos exames de detecção do câncer de próstata
}

\author{
A masculine question: knowing possible obstacles to the accomplishment of the tests of detection of prostate \\ cancer
}

Maria Cecília Leite de Moraes, ${ }^{1, a}$, Robson da Costa Oliveira ${ }^{1, \mathrm{~b}}$, Maria de Jesus Silva ${ }^{1, \mathrm{c}}$

\section{RESUMO}

Objetivos: Determinar se os homens realizam o exame para deteç̧ão do câncer de próstata e, desvelar as dificuldades para realiza-lo. Material e métodos: Estudo exploratório e transversal. Participaram 52 servidores do Tribunal de Justiça do Estado de São Paulo. Para a coleta de informações foi elaborada uma estória com perguntas atinentes ao assunto. Também foram colhidos os seguintes dados demográficos: idade, escolaridade, função e religião. A metodologia empregada para análise dos dados foi o Discurso do Sujeito Coletivo. Resultados: $75 \%$ dos indivíduos já realizou algum tipo de exame, o que indicou uma importante mudança no cenário masculino. Contudo, foi possível observar aspectos peculiares da masculinidade; entre os respondentes, apenas um explicitou a realização do exame de toque retal. Conclusões: Tabu, preconceito, vergonha e medo ainda são perigosos dados de realidade. Os profissionais e os serviços devem estar preparados para receber e acolher este público. A desmistificação é promoção de saúde.

PALAVRAS-CHAVE: Neoplasias da próstata, saúde do homem, diagnóstico precoce, educação em saúde, promoção de saúde. (Fonte: DeCS BIREME).

\section{SUMMARY}

Objectives: To determine if men undergo the examination for prostate cancer detection, and uncover the difficulties to perform it. Methods: Exploratory and transversal study. It was attended by 52 employees of the Court of Justice of the State of São Paulo. For the collection of information a story was elaborated with questions related to the subject. The following demographic data were also collected: age, education, function and religion. The methodology used to analyze the data was the Discourse of the Collective Subject. Results: $75 \%$ of the individuals had already performed some type of examination, which indicated an important change in the male scenario. However, it was possible to observe aspects peculiar to masculinity; Among respondents, only one made explicit the rectal examination. Conclusions: Taboo, prejudice, shame and fear are still dangerous reality data. Professionals and services should be prepared to receive and welcome this audience. Demystification is health promotion.

KEYWORDS: Prostate neoplasms, men's health, early diagnosis, health education, health promotion. (Source: MeSH NLM).

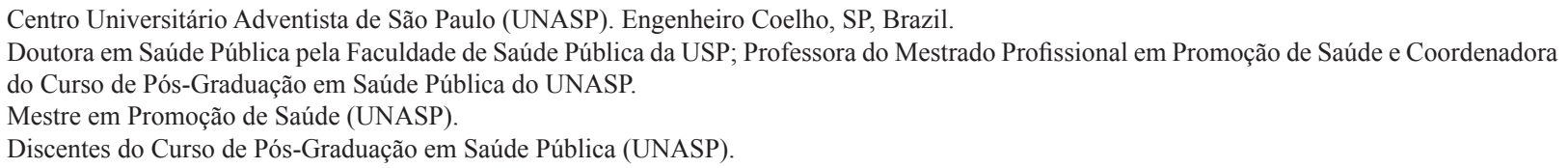




\section{INTRODUÇÃO}

A definição de gênero é crucial para a compreensão de atitudes e comportamentos humanos. Gênero não é sinônimo de sexo; o último determina aspectos biológicos, anatômicos e fisiológicos. Gênero indica características que padronizam o contraste entre feminino e masculino e, as associações que se constroem entre os dois grupos no quadro social (1).

Entre os indivíduos do gênero masculino, existe uma enorme dificuldade em aceitar suas vulnerabilidades, fator este relacionado a representação sócio cultural $(2,3)$. Em tempos mais recentes, a atenção à saúde desta população começa a ocupar um lugar de destaque na agenda sanitária (2). O cuidado relaciona-se as altas e maiores taxas de mortalidade (entre os homens) masculina em todas as faixas etárias, com aspectos específicos na morbimortalidade $(3,4)$.

Os estudos de morbidade masculina mostram que entre os anos de 2000 a 2007, houve um aumento de $100 \%$ nos casos de neoplasias (5). Destaca-se o câncer de próstata, sexto mais frequente no mundo, com cerca de $10 \%$ dos casos $(6,7)$.

O mais importante fator de risco para o desenvolvimento do câncer de próstata é a idade, 30\% dos casos são observados a partir dos 50 anos (8). Outros fatores de risco são: etnia (homens negros têm um risco significativamente aumentado) e histórico familiar $(8,9)$. Atualmente, reputa-se o estilo de vida como forte coadjuvante para o desenvolvimento da doença; dietas com alto teor calórico, ricas em gordura animal são fatores predisponentes. Vícios como tabagismo e etilismo, assim como, a obesidade e o sedentarismo potencializam os riscos para a patologia (5.10).

Ainda não se confirma a existência de prevenção para o câncer de próstata, o que se conhece é o rastreamento e o diagnóstico precoce (11). Contudo, a prematuridade diagnóstica oferece chances de cura que alcançam 90\%. É recomendado o exame preventivo que consiste na coleta de amostragem sanguínea para dosagem de antígeno prostático específico (PSA) e o toque retal (7). A maior parte dos estudos preconizam que os exames devem ser realizados, anualmente, a partir dos 50 anos de idade (12).

O grande desafio para a detecção precoce da doença parece estar na resistência dos homens com relação ao exame de toque retal. Para muitos, o exame aviltaria a masculinidade (6).
Considerando os pressupostos, o objetivo da pesquisa foi determinar se os homens realizam o exame para detecção do câncer de próstata e, desvelar as dificuldades para realiza-lo. Acredita-se que esta escuta contribui na capacitação de profissionais e investigadores da saúde quanto a abordagem do tema e, acolhimento para a execução do exame.

\section{MATERIAL E MÉTODOS}

Optou-se por uma pesquisa de abordagem quantiqualitativa, exploratória com delineamento transversal.

A amostra foi constituída por 52 servidores do Tribunal de Justiça do Estado de São Paulo, lotados em dez locais diferentes: fóruns, gabinetes de desembargadores, Palácio da Justiça e Secretaria da Área de Saúde do Tribunal de Justiça; todos no Município de São Paulo. A participação dos servidores foi aleatória, fazendo parte do grupo de investigação, apenas os que concordaram em atender ao convite, assinando o Termo de Consentimento Livre e Esclarecido. O documento para a coleta dos dados foi entregue, respondido e devolvido nos serviços.

A fim de caracterizar a amostra foram reunidos os seguintes dados demográficos: idade, cor, escolaridade, função, religião referida.

Para saber se os homens realizam o exame de detecção do câncer de próstata e, conhecer as dificuldades para efetivação do mesmo foi elaborada uma estória contemplando o tema e formuladas questões atinentes a ela. $\mathrm{O}$ artigo aborda três questões do estudo. Seguem-se a estória e as questões:

José e Antônio são amigos e colegas de trabalho há muitos anos.

Recentemente, perderam um colega Fernando, na faixa de 50 anos, com câncer de próstata.

Os três eram muito próximos. Após o trabalho conversam sobre o assunto.

José: - Foi triste o que aconteceu com o Fernando... Antônio: - É...

José: - Vamos ter excelentes lembranças, muitas estórias...

Antônio: - Será difícil...

(Minutos depois)

José: Já marquei meu exame

Antônio: - Credo!!!! Deus me livre!!!

José: Tomara que Deus te livre mesmo!!!

1. O que você achou da atitude de José? 
2. Você já fez o exame para saber se é portador do câncer da próstata?

3. Por que?

Para a análise dos dados das duas questões abertas foi utilizada a técnica elaborada por Lefèvre e colaboradores, o Discurso do Sujeito Coletivo (DSC) $(13,14)$. Este método permite a organização e averiguação dos principais elementos presentes nas respostas. Tabula os dados qualitativos de natureza verbal ou escrita obtidos nos depoimentos. É uma modalidade de apresentação de resultados das pesquisas qualitativas, que possuem depoimentos como matéria prima. Os depoimentos são apresentados em forma de discurso-síntese escritos na primeira pessoa do singular, procedimento que visa expressar o pensamento de uma coletividade, como se este grupo fosse o emissor de um discurso.

A estratégia fundamenta-se em selecionar, de cada resposta individual a uma questão Expressões-Chaves (EC), que são os trechos mais significativos da resposta. As Expressões-Chave constituem as Ideias Centrais (IC) que são a síntese do conteúdo discursivo declarados nas Expressões-Chave. Com o material destas ExpressõesChave das Ideias Centrais constroem-se discursos síntese, na primeira pessoa do singular, que são os DSC, onde o entendimento de um grupo aparece como se fosse um discurso individual.

A coleta de dados ocorreu no mês de agosto de 2016. A pesquisa foi aprovada pelo Comitê de Ética com parecer consubstanciado $\mathrm{n}^{\mathrm{o}} 1616856$.

\section{RESULTADOS}

Quanto a caracterização dos 52 sujeitos, destacase que um absteve-se de preencher todos os dados demográficos. Entretanto, sua participação foi contabilizada, pois o mesmo respondeu as questões específicas, aspecto fundamental para a investigação. A idade de 51 indivíduos variou entre 32 e 63 anos; a média foi 48,7 anos. Quanto a escolaridade 59,6\% possuíam nível superior completo e, 7,7\% incompleto; fator que mostra uma população diferenciada no âmbito educacional; dois indivíduos não responderam a esta pergunta. O mesmo aconteceu no quesito cor, onde $71,1 \%$ declararam-se brancos. No aspecto religioso $40,4 \%$ afirmaram ser católicos, 38,4\% não referiram ou se declararam sem religião e, $21,2 \%$ possuíam outras religiões.

A partir da primeira pergunta "O que você achou da atitude de José?" foi possível extrair 3 ideias centrais
(IC), que originaram os discursos do sujeito coletivo (DSC).

IC 1- É uma atitude positiva (96\%)

DSC: Atitude adequada, inteligente, responsável e preventiva. Demonstra conhecimento dos informes médicos. Geralmente esta atitude é impulsionada por fatores externos, como no caso; vendo a fatalidade ocorrida com seu amigo, tratou de se conscientizar e cuidar da saúde. Todos os homens deveriam fazer o mesmo, principalmente, após conhecerem pessoas que faleceram em decorrência da doença. A ação aponta para alguém esclarecido sobre os riscos e consequências inerentes ao câncer da próstata, que não se influencia pelos preconceitos. Antes tarde do que nunca, é preciso compreender que está protegendo a própria vida.

\section{IC 2- Evasivo (2\%)}

DSC: Nada explicativo...deveria explicar sobre exames que os homens devem fazer.

\section{IC3- Não respondeu (2\%)}

A resposta para a segunda pergunta "Você já fez exame para saber se é portador de câncer de próstata?" apontou que $75 \%$ dos homens já passaram pelo exame e, $25 \%$ ainda não.

A resposta para a terceira pergunta "Por que?" mostrou a justificativa para a realização ou não do exame. Entre os homens que afirmaram ter realizado o exame, todos se encaixaram na ideia central "autocuidado". A partir das respostas negativas foram localizadas 04 alegações que resultaram em 04 ideias centrais: "não se enquadra pela faixa etária", "relaxado", "preconceito" e, "subterfúgio". Entre os que ainda não passaram pelo exame, 8 (61\%) disseram estar fora da idade preconizada pelo protocolo. As ideias estão subsidiadas pelos respectivos discursos.

IC 1- (Sim) Autocuidado (96\%)

DSC: Sim, tenho consciência dos riscos envolvidos, faço pela qualidade de vida. Com 50 anos o médico sempre pede. Apesar da eficácia do PSA, sei que para haver confiabilidade no resultado, é necessário o exame de toque, às vezes até outros exames complementares. Sei que o histórico familiar pesa e, que a prevenção precoce aumenta a chance de cura.

IC 2- (Não). Não se enquadra (61\%)

DSC: Ainda não, pois não estou na faixa etária, mas sei da importância, tenho um caso na família. 
IC 3- (Não). Subterfúgio (23\%)

DSC: Ainda não, por causa da agenda pública para marcação de exame, mas estou providenciando, tenho casos na família, o médico pediu o ano passado.

IC 4- (Não). Relaxado (8\%)

DSC: Ainda não, mas pretendo fazer...é puro relaxo. Acho o exame importante e necessário.

\section{IC 5- (Não) Preconceito (8\%)}

DSC: Ainda não, pois o modo como é feito o mesmo é um pouco constrangedor.

\section{DISCUSSÃO}

Em referência a pergunta "O que você achou da atitude de José?" prevaleceu a ideia central da prevenção como "atitude positiva" (96\%), mostrando que os homens sabem da importância do exame. Mesmo diante desta compreensão, pesquisadores citam que ainda são poucos os indivíduos que realizam os exames preventivos (9). Ressalva-se que a ocorrência é comum contemplando inclusive sujeitos com altos níveis de escolaridade $(6,15)$. Estudos indicam que, no tocante ao câncer de próstata, os homens procuram os serviços de saúde quando as dores já são insuportáveis, ou ainda quando os sintomas impossibilitam o trabalho (6). A insistência para o cuidado de rotina deve ser uma das tônicas dos profissionais de saúde para esta população.

Quanto a segunda ideia central "evasiva", o discurso destacou uma associação com o desconhecimento. O respondente citou a ignorância, a falta de explicação quanto aos exames. Investigações corroboram esta lacuna, apontando a ausência de orientação adequada sobre o tema, incluso fatores de risco $(1,9)$. $O$ não saber constitui um forte quadro de vulnerabilidade.

A não resposta para a questão expressou significados encobertos tais como insegurança, medo de confrontar-se com uma dor real, ou ainda a vergonha que traduz noções simbólicas do universo masculino. Ao explorar o tema americanos e brasileiros assinalam estes aspectos $(16,17)$.

Setenta e cinco por cento dos entrevistados já passou pelos exames de detecção de câncer da próstata. O discurso do autocuidado foi expressivo revelando que, além da preocupação no que diz respeito a manutenção da saúde, há uma mudança de comportamento nos homens. Pesquisas anteriores citam a dificuldade do grupo em reconhecer suas necessidades de saúde (18) e, autores diversos ressalvam a falta de autocuidado de saúde entre os homens, com pretextos que variam desde as raízes culturais (19), até a própria conformação dos serviços (18). Foi interessante observar que um dos respondentes relatou não ter realizado o exame por conta da agenda do serviço público.

Neste estudo foi possível distinguir peculiaridades masculinas. Mesmo sem a pergunta sobre qual seria o exame já feito, o retorno alcançado mostrou-se interessante. Dentre 39 indivíduos que afirmaram a realização do exame, 29 (74\%) não explicitaram o exame; 7 declararam o exame de PSA, um referiu ter efetuado todos os exames e, apenas um expõe claramente ter efetuado o exame de toque retal. $\mathrm{O}$ não esclarecimento mostra importantes aspectos das singularidades masculinas, com silenciamentos que revelam proibições e profanações e, alimentam o imaginário coletivo do ser homem (20). Ainda, entre os pacientes que realizaram o exame de PSA, um destaca que não fez o exame de toque retal porque "não achou necessário".

O discurso presente na ideia central "não se enquadra" em função da faixa etária foi relatada por indivíduos entre idades de 32 a 47 anos. Mesmo sem haver discrepância, tal declaração foi inquietante já que o início para o rastreamento do câncer de próstata apresenta variações documentais, as orientações matizam-se desde 45 até os 50 anos $(6,21)$. A Sociedade Brasileira de Urologia preconiza que os exames devem ser feitos a partir dos 50 anos para a população geral e, aos 40 anos para os que possuem histórico familiar (20). Outros serviços orientam que o grupo mais vulnerável inicie o controle aos 45 anos (21). Ainda, há profissionais que solicitam exames a partir de quarenta anos.

A ideia central "relaxado" foi referenciada nas respostas e discursos. A negligência envolve questões culturais e dificuldade no enfrentamento de uma possível doença (19). "Estar doente não é compatível com o sujeito homem", tal concepção o desloca para o diagnóstico tardio, prognóstico ruim e, mudança na expectativa de vida (17). Vale destacar que a ideia está presente, mesmo entre indivíduos com formação médica. A pesquisa de Miranda et al (15), salienta o tema, constatando que os médicos se sentem confiantes em relação a sua saúde (16). É difícil compreender que parte dos profissionais, em idades preconizadas para o cuidado, jamais tenham realizado práticas preventivas. São pessoas conhecedoras do assunto, possuem acesso fácil e garantido aos serviços de diagnóstico clínico e 
complementar. O constructo reforça a importância do entendimento e estudos de gênero.

Torna-se digno de nota que o discurso "preconceito" tenha sido declarado por $2 \%$ da população amostral. É um fator real e importante entre sujeitos de sexo masculino. Existe dificuldade em aceitar o método de diagnóstico toque retal, que reflete o imaginário social quanto a identidade sexual do grupo. Diz respeito a invasão de um espaço próprio e pessoal, percebida como uma parte (o ânus) que não deve ser explorada. Há uma normatização tácita do que pode ou não ser mostrado ou tocado, mesmo quando o indivíduo compreende tratar-se de tabu (20). Enfatiza-se, uma vez mais, que o preconceito, a vergonha e o medo são os principais motivos para a negligência no cuidado com a próstata, considerados como fatores impeditivos para a realização do exame (17). Desnudar mitos quanto ao exame deve ser trabalhado sempre e de forma contínua.

Mesmo diante do incomodo e preconceito constituídos culturalmente, o acompanhamento da saúde como forma de prevenção e manutenção da vida vem se transformando em uma "conquista vitoriosa". Os aspectos das peculiaridades masculinas foram enfatizados no silenciamento quanto ao tipo de exame e a clara afirmação de constrangimento. $\mathrm{O}$ enfoque cultural e, a construção simbólica acerca do procedimento continuam a ser potentes inibidores. Compreende-se que o desconforto é uma sensação real, entretanto a prevenção é necessária. A conscientização do grupo e da sociedade, no que diz respeito aos exames é um território a ser semeado sistematicamente pois, o zelo pela saúde muda o cenário social. A educação em saúde, especialmente, voltada para os homens e, os cuidados constantes são temas que devem ser tratados em diferentes contextos destacando-se casa, escola e trabalho. Os profissionais têm um papel fundamental que passa pela compreensão das singularidades do gênero, acolhimento e na própria condução do exame, envolvendo empatia e ambiência. A valorização de um espaço para o homem nos diversos serviços deve ser uma realidade. Os projetos campanhistas não são a solução, pois com certeza o problema não está na falta de informação, mas no uso desta a favor do cuidado. Mitos, crendices, prejulgamentos, vergonha e medo, ainda são perigosos dados de realidade. Compreensão, educação e reversão de tabus são os desafios. Desmitificação é promoção de saúde e, os programas continuados são a luta para este tempo.

\section{Declaração de financiamento y de conflitos de interesse:}

A pesquisa não recebeu qualquer tipo de patrocínio e/ ou financiamento.

Não há conflito de interesses.

\section{Contribuição de autoria:}

MCLM: Concepção e elaboração da pesquisa, análise e interpretação dos dados, redação do artigo; RCO: análise e elaboração dos dados e MSJ: tabulação e interpretação de dados.

\section{Correspondência:}

Maria Cecília Leite de Moraes

Rua Manoel Dutra 539, São Paulo, CEP 01328-010.

E-mail: leimo7@hotmail.com

\section{REFERENCIAS BIBLIOGRÁFICAS}

1. Barata RB. Relações de gênero e saúde. In: Como e por que as desigualdades sociais fazem mal à saúde. Rio de Janeiro: Editora Fiocruz, 2009 p.73.

2. Carrara S, Russo JA, Faro L. A política de atenção à saúde do homem no Brasil: os paradoxos da medicalização do corpo masculino. Physis (Rio J.). 2009; 19(3):659-678.

3. Laurenti R, Jorge MHPM, Gotlieb SLD. Perfil epidemiológico da morbi-mortalidade masculina. Ciênc saúde coletiva. 2005; 10(1):35-46.

4. Figueiredo WS. Assistência à saúde dos homens: um desafio para os serviços de atenção primária. Ciênc saúde coletiva. 2005; 10(1):105-9.

5. Tonon TCA, Schoffen JPF. Câncer de próstata: uma revisão da literatura. Saud Pesq. 2009; 2(3):403-410.

6. de Souza LM, Silva MP, de Souza Pinheiro I. Um toque na masculinidade: a prevenção do câncer de próstata em gaúchos tradicionalistas. Rev Gaúcha Enferm. 2011; 32(1):151.

7. Vieira CG, Araújo WS, Vargas DRM. O homem e o câncer de próstata: Prováveis reações diante de um possível diagnóstico. Revista Científica do ITPAC. 2012; 5(1):1-9.

8. Dornas MC, Júnior JADR, Figueiredo e Filho RT, Carrerette FB, Damião R. Câncer de próstata. Revista HUPE. 2008; 7:100-107.

9. Gonçalves IR, Padovani C, Popim RC. Caracterização epidemiológica e demográfica de homens com câncer de próstata. Ciênc saúde coletiva. 2008;13(4):13371342.

10. Lima ACF, da Silva KVM, Caetano JÁ, de Lima MA, de Andrade LM. Conhecimento dos trabalhadores de uma universidade privada sobre a prevenção do 
câncer de próstata. Cogitare Enferm. 2007; 12(4):460465.

11. Oliveira JIM, Popov DCS. Exame preventivo do câncer de próstata: impressões e sentimentos. Rev Enferm UNISA. 2012; 13(1):13-20.

12. Sociedade Brasileira de Urologia. Novembro Azul conscientiza sobre câncer de próstata. Rio de Janeiro: Sociedade Brasileira de Urologia; 2015.

13. Lefèvre F, Lefèvre AMC, Teixeira JJV. Os novos instrumentos no contexto da pesquisa qualitativa. Lefèvre F, Lefèvre AMC, Teixeira JJV, organizadores. O discurso do sujeito coletivo: uma nova abordagem metodológica em pesquisa qualitativa. Caxias do Sul: EDUCS; 2000. p. 11-35.

14. Lefevre F, Lefevre, AMC. O sujeito coletiv O sujeito coletivo que fala o que fala. Interface (Botucatu). 2006; 10(20):517-24.

15. Miranda PSC, Côrtes MCJW, Martins ME, Chaves PC, Santarosa RC. Práticas de diagnóstico precoce de câncer de próstata entre professores da faculdade de medicina-UFMG. Rev Assoc Med Bras. 2004; 50(3):272-5.
16. Amorim VMSL, Barros MBDA, César CLG, Goldbaum M, Carandina L, Alves MCGP. Fatores associados à realização dos exames de rastreamento para o câncer de próstata: um estudo de base populacional. Cad saúde pública. 2011; 27(2):347356.

17. Silva L, Costa CMA, Martins ERC, Francisco MTR, Marta CB. Fatores impeditivos para o exame preventivo do câncer de próstata: visão masculina. Rev Saúde Corpo Ambiente e Cuidado. 2013; 1(1):143156.

18. Silva PADS, Furtado MDS, Guilhon AB, Souza NVDO, David HMSL. A saúde do homem na visão dos enfermeiros de uma unidade básica de saúde. Esc Anna Nery. 2012; 16(3):561-8.

19. Meirelles RM, Hohl A. Saúde masculina: tão negligenciada, principalmente pelos homens. Arq bras endocrinol metab. 2009; 53(8):899-900.

20. Gomes R, do Nascimento EF, de Araújo, FC. Por que os homens buscam menos os serviços. Cad saúde pública. 2007; 23(3):565-574.

21. Medeiros APD, Menezes MDFBD, Napoleão AA. Fatores de risco e medidas de prevenção do câncer de próstata: subsídios para a enfermagem. Rev bras enferm. 2011; 64(2):385-388.

Recibido: 23/03/2017

Aceptado: 19/08/2017 